\title{
ORIGINAL ARTICLE SEVERE MITRAL REGURGITATION AFTER PMBV? COMPARISON BETWEEN INOUE AND MULTI-TRACK TECHNIQUES
}

\author{
Faiza Farooq ${ }^{1}$, Ali Ammar', Iram Jehan Balouch², Ayaz Mir¹, Atif Sher Muhammad', \\ Syed Alishan ul Haq ${ }^{3}$, Tahir Saghir ${ }^{1}$, Tariq Ashraf ${ }^{4}$, Jawaid Akbar Sial' ${ }^{1}$, Naveedullah \\ Khan ${ }^{1}$
}

${ }^{1}$ National Institute of Cardiovascular Diseases, Karachi, Pakistan, ${ }^{2}$ National Institute of Cardiovascular Diseases, Hyderabad, Pakistan, ${ }^{3}$ Peshawar Institute of Cardiology, Peshawar, Pakistan, ${ }^{4}$ Karachi Institute of Heart Diseases, Karachi, Pakistan

Objectives: To compare the frequency of severe mitral regurgitation after percutaneous mitral balloon valvuloplasty (PMBV) via Inoue balloon and multi-track balloon technique in our population.

Methodology: In this retrospective observational study which was conducted at a tertiary care cardiac center of Karachi, Pakistan between 2015 and 2020 on Hospital registry of PMBV patients. Data were categorized in to two groups, Inoue balloon or multi-track balloon technique. Post procedure echocardiographic and catheterization parameters and in-hospital outcomes and complications, including severe MR, were compared between two groups.

Results: Out of 470 PMBV procedures, 286 (60.9\%) were performed with multi-track and 184 $(39.1 \%)$ with Inoue balloon. Improvement in mitral value area was significantly higher with multi-track as compared to Inoue balloon $(0.66 \pm 0.31 \mathrm{~cm} 2$ vs. $0.56 \pm 0.29 \mathrm{~cm} 2 ; \mathrm{p}<0.001)$. Severe MR was not significant, 3.5\% (10/286) vs. $4.3 \%(8 / 184) ; p=0.639$ for multi-track and Inoue balloon. One patient in Inoue balloon group and two patients in multi-track group required emergency valve surgery. Stroke was observed in two patients of multi-track group and two patients from the same group developed tamponade. No in-hospital mortality was observed.

Conclusion: Post-procedure severe MR is a significant and frequent complication. Rate of post procedure severe MR are similar for PMBV via Inoue balloon and multi-track balloon. Both methods are equally effective with equal success rate.

Keywords: rheumatic heart disease; rheumatic fever; mitral stenosis; percutaneous mitral balloon valvuloplasty; mitral regurgitation; Inoue balloon; multi-track balloon

\begin{abstract}
Citation: Farooq F, Ammar A, Balouch IJ, Mir A, Muhammad AS, Haq SA, Saghir T, Ashraf T, Sial JA, Khan N. Severe Mitral Regurgitation after PMBV? Comparison between Inoue and Multi-track Techniques. Pak Heart J. 2021;54(04):333-338. DOI: https://doi.org/10.47144/phj.v54i4.2133
\end{abstract}

\section{INTRODUCTION}

Prevalence of rheumatic heart disease (RHD) is slim to none in developed nations as a result of effective preventive measures such as reduction in exposure to the group-A streptococcus (GAS) bacterial infection and use of primary prophylaxis. ${ }^{1}$ Conversely, it remains a major challenge in low- and -middle income, underdeveloped and developing nations. It remains one of the leading cardiovascular disorder in young population, and major cause of significant premature mortality and morbidity. ${ }^{1-3}$ Valvular damage in early years of life due to abnormal immune response to GAS infection remains the major cause of RHD. According to World Health Organization (WHO), RHD is the most common acquired cardiovascular disorder in young ( $<25$ years of age) population with around 291 thousand associated annual fatalities with major share of low- and -middle income countries (LMCs). ${ }^{4}$ The Western Pacific regions, South-East Asian, and African counties are the highly affected regions with persistently high incidence for RHD with nearly $84 \%$ of the reported cases and $80 \%$ of mortalities in the year $2015 .{ }^{5}$ It has not only have significant impact on the economy of the country but also has devastating effects on productive years of adults as well as children. Various environmental and socioeconomic factors are known to contribute to the increased incidence of RHD in LMCs, such as lack of access and inadequate healthcare facilities, poverty, malnutrition, overcrowdings, and poor living conditions. ${ }^{6}$

Mitral stenosis (MS), a progressive reduction of mitral valve area after acute rheumatic fever (RF), accounts for around one fourth of the total cases of RHD. Yearly reduction in mitral valve (MV) area varies from 0.1 to $0.3 \mathrm{~cm}^{2}$ depending on the population under study. Patients in LMCs are observed to present at much younger age as compared to high income countries patients. ${ }^{7,8}$ They usually remains symptom free until MV area reduces to $1.5 \mathrm{~cm}^{2}$. This is when appropriate management become compulsory and if left untreated patients may develop severe life-threatening conditions such as stroke, atrial fibrillation, pulmonary 
embolism, pulmonary oedema, or pulmonary hypertension. ${ }^{7}$ Along with secondary prophylaxis to prevent recurrence of acute RF, mechanical management approaches (either surgical or percutaneous) have proved effective in improving outcome of these patients to a greater extent. ${ }^{7}$

Percutaneous mitral balloon valvuloplasty (PMBV), also known as percutaneous transvenous mitral commissurotomy (PTMC), is treatment of choice with good outcomes for symptomatic patients of severe mitral stenosis. It is class I indication for symptomatic patients with suitable valve morphology, MV area of $\leq 1.5 \mathrm{~cm}^{2}$, and absence of moderate-to-severe mitral regurgitation and left atrial thrombus.9, 10 Various PMBV techniques have evolved over the time such as metallic valvotome, multi-track balloon, and Inoue balloon. Later two methods are more commonly in practice in our part of the world. ${ }^{11}$ Despite advancements in techniques, expertise and years of experience, mitral regurgitation (MR) after PMBV procedure remains the main procedure-related complication and varying rates have been reported depending on techniques, operators expertise, and valve anatomy. ${ }^{12}$

Both multitrack balloon and Inoue balloon are in practice in our setting. However, data regarding post PMBV severe MR complication for both techniques are very limited for our population. Therefore, objective of this analysis was to compare the frequency and severity of MR after PMBV with Inoue balloon versus multi-track double balloon techniques.

\section{METHODOLOGY}

This retrospective observational study was conducted at the National Institute of Cardiovascular Diseases (NICVD), Karachi, Pakistan. Our center is the largest cardiac care and training center of the country with an average yearly volume of 1200 PMBV procedures performed by 12 trained consultant cardiologists.

Approval from the ethical review committee of the institution was taken to access the institutional database for this study. In order to minimize the intraoperator biasness and time dependent technical variations of PMBV procedure, we have decided to include data of the procedures performed by the 4 equally experienced senior Interventional cardiologist from the year 2015 to 2020 .

Clinical and procedure related data of consecutive PMBV procedures performed for the patients of either gender with severe MS via Inoue balloon or multitrack double balloon were extracted from prospectively maintained institutional database. Patients with left atrial (LA) thrombus, severe MR at baseline echocardiographic assessment, and required cardiac surgery due to severe aortic, tricuspid, or coronary disease were excluded from this analysis. Extracted data comprises of baseline demographic characteristics (such as age, gender, ECG findings, history related to cerebrovascular accident (CVA), embolic event, known coronary artery diseases, pregnancy, and baseline echocardiographic assessment such as left ventricular (LV) dysfunction, left atrial (LA) smoke, Wilkin score, mitral regurgitation (MR), and tricuspid regurgitation (TR)), and procedure related data such as pre- and postprocedure echocardiographic parameters (such as pulmonary artery (PA) pressure, mitral valve (MV) area, and left atrio-ventricular gradient), catheterization data (such as left ventricular enddiastolic pressure (LVEDP), LA mean pressure, and LA mean gradient), and in-hospital complications (severity of MR and severity of TR) and outcomes (procedure success, stroke, tamponed, emergency valve surgery, and mortality). Patient's data with missing variables, incomplete or terminated procedure or table death during procedure was not included in final data.

Extracted data were analyzed with the help of IBM SPSS version 21, data were categorized into two groups; Inoue balloon and multi-track double balloon, continuous variables were expressed as mean \pm standard deviations (SD) and categorical variables will be expressed as frequency and percentages. Two groups were compared by applying chi-square test for categorical variables and independent sample t-test for continuous variables. Pre and post procedure differences in echocardiographic parameters (such as PA pressure, MV area, and left atrioventricular gradient) and catheterization parameters (such as LVEDP, LA mean pressure, and LA mean gradient) were assessed by applying paired-sample t-test. Pvalue of $\leq 0.05$ was considered significant in all performed analysis.

\section{RESULTS}

Data of total of 470 PMBV procedures performed by selected operators in defined period were evaluated in this study, $286(60.9 \%)$ of these procedure were performed with multi-track balloon technique and remaining $184(39.1 \%)$ of the procedures were performed via Inoue balloon technique. Majority $(74.7 \%)$ of the procedure were performed in female patients, mean age at the time of procedure was $31.8 \pm$ 10.7 years with $16.2 \%$ (76) of patients above 40 years of age. Mean Wilkin score was $7.33 \pm 1.46$. Associated mild valvular diseases were present in $31.7 \%$ (149) of the patients and $33.6 \%$ (158) of the patients have LV dysfunction at baseline. 
Comparative assessment of multi-track and Inoue balloon group showed significant difference in mean age $(30.57 \pm 10.04$ years vs. $33.71 \pm 11.43$ years; $\mathrm{p}=0.002)$, body weight $(52.14 \pm 12.25 \mathrm{~kg}$ vs. $55.32 \pm$ $12.9 \mathrm{~kg} ; \mathrm{p}=0.008)$, Wilkin score $(7.18 \pm 1.46$ vs. 7.58 $\pm 1.42 ; \mathrm{p}=0.004)$, presence of associated valvular disease (36\% (103/286) vs. $25 \%(46 / 184) ; \mathrm{p}=0.012)$, and presence of LA smoke (11.9\% (34/286) vs. $25.5 \%$ (47/184); $<<0.001)$ for multi-track and Inoue balloon group respectively. Baseline characteristics of the patients stratified by procedure technique are presented in Table 1 .

Table 1: Baseline characteristics of the patients stratified by procedure technique

\begin{tabular}{|c|c|c|c|c|}
\hline \multirow{2}{*}{ Characteristics } & \multirow{2}{*}{ Total } & \multicolumn{2}{|c|}{$\begin{array}{l}\text { Procedure } \\
\text { Technique }\end{array}$} & \multirow{2}{*}{ P-value } \\
\hline & & \begin{tabular}{c|}
$\begin{array}{c}\text { Multi- } \\
\text { track }\end{array}$ \\
\end{tabular} & \begin{tabular}{|c|}
$\begin{array}{c}\text { Inoue } \\
\text { balloon }\end{array}$ \\
\end{tabular} & \\
\hline Total (N) & 470 & $\begin{array}{c}286 \\
(60.9 \%)\end{array}$ & $\begin{array}{c}184 \\
(39.1 \%)\end{array}$ & - \\
\hline \multicolumn{5}{|l|}{ Gender } \\
\hline Female & $\begin{array}{l}74.7 \% \\
(351)\end{array}$ & $\begin{array}{l}73.8 \% \\
(211)\end{array}$ & $\begin{array}{l}76.1 \% \\
(140)\end{array}$ & \multirow{2}{*}{0.574} \\
\hline Male & $\begin{array}{c}25.3 \% \\
(119) \\
\end{array}$ & $\begin{array}{c}26.2 \% \\
(75) \\
\end{array}$ & $\begin{array}{c}23.9 \% \\
(44) \\
\end{array}$ & \\
\hline Age (years) & $\begin{array}{c}31.8 \pm \\
10.7\end{array}$ & $\begin{array}{c}30.57 \pm \\
10.04\end{array}$ & $\begin{array}{c}33.71 \pm \\
11.43\end{array}$ & $0.002 *$ \\
\hline$\leq 40$ years & $\begin{array}{c}83.8 \% \\
(394)\end{array}$ & $86 \%$ (246) & $\begin{array}{c}80.4 \% \\
(148)\end{array}$ & \multirow{2}{*}{0.109} \\
\hline$>40$ years & $\begin{array}{l}16.2 \% \\
(76)\end{array}$ & $14 \%(40)$ & $\begin{array}{c}19.6 \% \\
(36)\end{array}$ & \\
\hline Weight (kg) & $\begin{array}{c}53.39 \pm \\
12.59 \\
\end{array}$ & $\begin{array}{c}52.14 \pm \\
12.25 \\
\end{array}$ & $\begin{array}{c}55.32 \pm \\
12.9\end{array}$ & $0.008^{*}$ \\
\hline Height (cm) & \begin{tabular}{|c|}
$155.69 \pm$ \\
10.17 \\
\end{tabular} & $\begin{array}{c}155.66 \pm \\
10.53\end{array}$ & $\begin{array}{c}155.74 \pm \\
9.6\end{array}$ & 0.939 \\
\hline \multicolumn{5}{|l|}{ Cardiac Rhythm } \\
\hline Sinus & $\begin{array}{l}87.2 \% \\
(410)\end{array}$ & $\begin{array}{l}87.1 \% \\
(249)\end{array}$ & $\begin{array}{c}87.5 \% \\
(161)\end{array}$ & \multirow{2}{*}{0.890} \\
\hline Afib/Flutter & $\begin{array}{c}12.8 \% \\
(60)\end{array}$ & $\begin{array}{c}12.9 \% \\
(37)\end{array}$ & $\begin{array}{c}12.5 \% \\
(23)\end{array}$ & \\
\hline $\begin{array}{l}\text { Associated mild } \\
\text { valvular disease }\end{array}$ & $\begin{array}{l}31.7 \% \\
(149)\end{array}$ & $36 \%(103)$ & $25 \%(46)$ & $0.012 *$ \\
\hline $\begin{array}{l}\text { Prior } \\
\text { cerebrovascular } \\
\text { accident }\end{array}$ & $1.3 \%(6)$ & $1 \%(3)$ & $1.6 \%(3)$ & 0.584 \\
\hline $\begin{array}{l}\text { Prior embolic } \\
\text { episode }\end{array}$ & $0.9 \%(4)$ & $0.7 \%(2)$ & $1.1 \%(2)$ & 0.655 \\
\hline $\begin{array}{l}\text { Known coronary } \\
\text { artery diseases }\end{array}$ & $1.9 \%(9)$ & $1 \%(3)$ & $3.3 \%(6)$ & 0.088 \\
\hline Pregnancy & $\begin{array}{c}2.6 \% \\
(12)\end{array}$ & $3.1 \%(9)$ & $1.6 \%(3)$ & 0.309 \\
\hline Left atrial smoke & $\begin{array}{c}17.2 \% \\
(81)\end{array}$ & $\begin{array}{c}11.9 \% \\
(34)\end{array}$ & $\begin{array}{c}25.5 \% \\
(47)\end{array}$ & $<0.001 *$ \\
\hline Wilkin score & $\begin{array}{c}7.33 \pm \\
1.46\end{array}$ & $\begin{array}{c}7.18 \pm \\
1.46\end{array}$ & $\begin{array}{c}7.58 \pm \\
1.42\end{array}$ & $0.004 *$ \\
\hline$<8$ & $\begin{array}{c}49.8 \% \\
(234)\end{array}$ & $\begin{array}{c}49.7 \% \\
(142)\end{array}$ & $50 \%(92)$ & 0.941 \\
\hline 8 to 9 & $\begin{array}{c}37.9 \% \\
(178)\end{array}$ & $\begin{array}{c}41.3 \% \\
(118)\end{array}$ & $\begin{array}{c}32.6 \% \\
(60)\end{array}$ & 0.059 \\
\hline 10 to 11 & $\begin{array}{c}12.3 \% \\
(58)\end{array}$ & $9.1 \%(26)$ & $\begin{array}{c}17.4 \% \\
(32)\end{array}$ & $0.008^{*}$ \\
\hline
\end{tabular}

\begin{tabular}{|c|c|c|c|c|}
\hline \begin{tabular}{|l|} 
Left ventricular \\
dysfunction
\end{tabular} & $\begin{array}{c}33.6 \% \\
(158) \\
\end{array}$ & $\begin{array}{c}34.3 \% \\
(98) \\
\end{array}$ & $\begin{array}{c}32.6 \% \\
(60)\end{array}$ & 0.711 \\
\hline \multicolumn{5}{|c|}{ Pre-procedure mitral regurgitation (MR) } \\
\hline None & $\begin{array}{c}49.6 \% \\
(233) \\
\end{array}$ & $57 \%(163)$ & $38 \%(70)$ & $<0.001 *$ \\
\hline Mild & $\begin{array}{c}48.3 \% \\
(227)\end{array}$ & $\begin{array}{c}41.6 \% \\
(119)\end{array}$ & $\begin{array}{c}58.7 \% \\
(108)\end{array}$ & $<0.001 *$ \\
\hline Moderate & $\begin{array}{l}2.1 \% \\
(10)\end{array}$ & $1.4 \%(4)$ & $3.3 \%(6)$ & 0.172 \\
\hline \begin{tabular}{|l|} 
Severe \\
\end{tabular} & $0 \%(0)$ & $0 \%(0)$ & $0 \%(0)$ & - \\
\hline \multicolumn{5}{|c|}{ Pre-procedure tricuspid regurgitation (TR) } \\
\hline None & $\begin{array}{c}20.6 \% \\
(97) \\
\end{array}$ & $\begin{array}{c}21.3 \% \\
(61) \\
\end{array}$ & $\begin{array}{c}19.6 \% \\
(36) \\
\end{array}$ & 0.645 \\
\hline Mild & $\begin{array}{c}23.2 \% \\
(109) \\
\end{array}$ & $\begin{array}{c}18.9 \% \\
(54) \\
\end{array}$ & $\begin{array}{c}29.9 \% \\
(55) \\
\end{array}$ & $0.006^{*}$ \\
\hline Moderate & $\begin{array}{c}38.5 \% \\
(181)\end{array}$ & $\begin{array}{l}40.6 \% \\
(116)\end{array}$ & $\begin{array}{c}35.3 \% \\
(65) \\
\end{array}$ & 0.255 \\
\hline Severe & $\begin{array}{c}17.7 \% \\
(83)\end{array}$ & \begin{tabular}{|c|}
$19.2 \%$ \\
$(55)$
\end{tabular} & $\begin{array}{c}15.2 \% \\
(28)\end{array}$ & 0.265 \\
\hline \multicolumn{5}{|c|}{ Dilatation procedure total number of opening } \\
\hline $\begin{array}{ll} \\
\end{array}$ & $\begin{array}{c}18.1 \% \\
(85)\end{array}$ & \begin{tabular}{|c|}
$12.2 \%$ \\
$(35)$
\end{tabular} & \begin{tabular}{|c|}
$27.2 \%$ \\
$(50)$ \\
\end{tabular} & $<0.001 *$ \\
\hline 2 & $\begin{array}{c}58.5 \% \\
(275)\end{array}$ & $\begin{array}{c}60.5 \% \\
(173)\end{array}$ & $\begin{array}{c}55.4 \% \\
(102)\end{array}$ & 0.278 \\
\hline 3 & $\begin{array}{l}23.4 \% \\
(110)\end{array}$ & $\begin{array}{c}27.3 \% \\
(78)\end{array}$ & $\begin{array}{c}17.4 \% \\
(32)\end{array}$ & $0.014 *$ \\
\hline
\end{tabular}

Pre- and post-procedure changes in echocardiographic and catheterization parameters stratified by procedure technique are presented in Table 2. A significant reduction of $23.89 \pm 15.38 \mathrm{mmHg}$ was observed in post procedure PA pressure. Reduction in PA pressure was same in both multi-track and Inoue balloon group with reduction of $23.73 \pm 15.74 \mathrm{mmHg}$ vs. $24.14 \pm$ $14.85 \mathrm{mmHg} ; \mathrm{p}=0.776$. Improvement in post procedure mitral value area $(0.66 \pm 0.31 \mathrm{~cm} 2$ vs. 0.56 $\pm 0.29 \mathrm{~cm} 2 ; \mathrm{p}<0.001)$ as compared to Inoue balloon group, similarly, reduction in left atrioventricular gradient $(8.96 \pm 5.01 \mathrm{mmHg}$ vs. $7.79 \pm 4.52 \mathrm{mmHg}$; $\mathrm{p}=0.010)$ was significantly higher in multi-track group as compared to Inoue balloon group. A significant improvement in post procedure catheterization parameters (such as LVEDP, LA mean pressure, and LA mean gradient) was observed in both multi-track and Inoue balloon group.

Table 2: Evaluation of pre- and post-procedure echocardiographic and catheterization parameters stratified by procedure technique

\begin{tabular}{|c|c|c|c|c|}
\hline \multirow{2}{*}{ Parameters } & \multirow{2}{*}{ Total } & \multicolumn{2}{|c|}{$\begin{array}{l}\text { Procedure } \\
\text { Technique } \\
\end{array}$} & \multirow{2}{*}{ P-value } \\
\hline & & $\begin{array}{l}\text { Multi- } \\
\text { track }\end{array}$ & \begin{tabular}{|c|} 
Inoue \\
balloon
\end{tabular} & \\
\hline Total (N) & 470 & 286 & \begin{tabular}{|l|}
184 \\
\end{tabular} & - \\
\hline \multicolumn{5}{|c|}{ Echocardiography parameters } \\
\hline \multicolumn{5}{|c|}{ Pulmonary artery pressure (mmHg) } \\
\hline Pre procedure & $\begin{array}{c}63.07 \pm \\
21.28\end{array}$ & $\begin{array}{c}64.13 \pm \\
20.57\end{array}$ & \begin{tabular}{|c|}
$61.41 \pm$ \\
22.29
\end{tabular} & 0.177 \\
\hline
\end{tabular}




\begin{tabular}{|c|c|c|c|c|}
\hline Post procedure & $\begin{array}{c}39.18 \pm \\
17.11\end{array}$ & $\begin{array}{c}40.4 \pm \\
16.44\end{array}$ & \begin{tabular}{|l|}
$37.27 \pm$ \\
17.99
\end{tabular} & 0.053 \\
\hline Improvement & \begin{tabular}{|c|}
$-23.89 \pm$ \\
15.38
\end{tabular} & \begin{tabular}{|c|}
$-23.73 \pm$ \\
15.74
\end{tabular} & \begin{tabular}{|c|}
$-24.14 \pm$ \\
14.85
\end{tabular} & 0.776 \\
\hline \begin{tabular}{|l|}
$\begin{array}{l}\text { Pre-post comparison } \\
\text { (p-value) }\end{array}$ \\
\end{tabular} & $<0.001 *$ & $<0.001 *$ & $<0.001 *$ & - \\
\hline \multicolumn{5}{|l|}{ Valve area $\left(\mathrm{cm}^{2}\right)$} \\
\hline Pre procedure & $\begin{array}{c}0.96 \pm \\
0.2\end{array}$ & $0.98 \pm 0.2$ & $\begin{array}{c}0.93 \pm \\
0.2\end{array}$ & $0.007 *$ \\
\hline Post procedure & $\begin{array}{c}1.58 \pm \\
0.3\end{array}$ & $\begin{array}{c}1.64 \pm \\
0.29 \\
\end{array}$ & $\begin{array}{c}1.49 \pm \\
0.3\end{array}$ & $<0.001^{*}$ \\
\hline Improvement & $\begin{array}{c}0.62 \pm \\
0.31\end{array}$ & $\begin{array}{c}0.66 \pm \\
0.31\end{array}$ & $\begin{array}{c}0.56 \pm \\
0.29\end{array}$ & $<0.001^{*}$ \\
\hline $\begin{array}{l}\text { Pre-post comparison } \\
\text { (p-value) }\end{array}$ & $<0.001 *$ & $<0.001 *$ & $<0.001 *$ & - \\
\hline \multicolumn{5}{|c|}{ Left atrioventricular peak gradient $(\mathrm{mmHg})$} \\
\hline Pre procedure & \begin{tabular}{|c|}
$15.41 \pm$ \\
4.85 \\
\end{tabular} & \begin{tabular}{|c|}
$15.61 \pm$ \\
4.94 \\
\end{tabular} & $\begin{array}{c}15.1 \pm \\
4.7\end{array}$ & 0.266 \\
\hline Post procedure & $\begin{array}{c}6.9 \pm \\
4.01 \\
\end{array}$ & $\begin{array}{c}6.64 \pm \\
3.85\end{array}$ & $\begin{array}{c}7.31 \pm \\
4.23\end{array}$ & 0.081 \\
\hline Improvement & $\begin{array}{c}-8.5 \pm \\
4.85\end{array}$ & $\begin{array}{c}-8.96 \pm \\
5.01\end{array}$ & \begin{tabular}{|l|}
$-7.79 \pm$ \\
4.52
\end{tabular} & $0.010^{*}$ \\
\hline $\begin{array}{l}\text { Pre-post comparison } \\
\text { (p-value) }\end{array}$ & $<0.001 *$ & $<0.001 *$ & $<0.001 *$ & - \\
\hline \multicolumn{5}{|c|}{\begin{tabular}{|l|} 
Catheterization parameters \\
\end{tabular}} \\
\hline \multicolumn{5}{|c|}{ Left ventricular end-diastolic pressure (mmHg) } \\
\hline Pre procedure & \begin{tabular}{|c|}
$13.53 \pm$ \\
4.09
\end{tabular} & \begin{tabular}{|c|}
$13.69 \pm$ \\
3.81
\end{tabular} & \begin{tabular}{|c|}
$13.28 \pm$ \\
4.49
\end{tabular} & 0.291 \\
\hline Post procedure & $\begin{array}{c}15.13 \pm \\
5.51\end{array}$ & $\begin{array}{c}15.69 \pm \\
5.42\end{array}$ & $\begin{array}{c}14.27 \pm \\
5.55\end{array}$ & $0.006^{*}$ \\
\hline Improvement & $\begin{array}{l}1.6 \pm \\
5.06 \\
\end{array}$ & $\begin{array}{c}2.00 \pm \\
4.95 \\
\end{array}$ & $\begin{array}{c}0.99 \pm \\
5.17 \\
\end{array}$ & $0.034 *$ \\
\hline $\begin{array}{l}\text { Pre-post comparison } \\
\text { (p-value) }\end{array}$ & $<0.001 *$ & $<0.001 *$ & $0.010^{*}$ & - \\
\hline \multicolumn{5}{|c|}{ Left atrial mean pressure (mmHg) } \\
\hline Pre procedure & \begin{tabular}{|c|}
$29.26 \pm$ \\
6.97 \\
\end{tabular} & \begin{tabular}{|c|}
$30.18 \pm$ \\
6.81 \\
\end{tabular} & $\begin{array}{c}27.84 \pm \\
7\end{array}$ & $<0.001^{*}$ \\
\hline Post procedure & $\begin{array}{c}19.04 \pm \\
6.92 \\
\end{array}$ & \begin{tabular}{|c|}
$19.63 \pm$ \\
6.34 \\
\end{tabular} & $\begin{array}{c}18.13 \pm \\
7.66 \\
\end{array}$ & $0.022 *$ \\
\hline Improvement & $\begin{array}{c}-10.22 \pm \\
7.81 \\
\end{array}$ & \begin{tabular}{|c|}
$-10.55 \pm$ \\
8.22 \\
\end{tabular} & $\begin{array}{c}-9.71 \pm \\
7.11 \\
\end{array}$ & 0.252 \\
\hline $\begin{array}{l}\begin{array}{l}\text { Pre-post comparison } \\
\text { (p-value) }\end{array} \\
\end{array}$ & $<0.001 *$ & $<0.001 *$ & $<0.001 *$ & - \\
\hline \multicolumn{5}{|c|}{ Left atrial mean gradient $(\mathrm{mmHg})$} \\
\hline Pre procedure & \begin{tabular}{|c|}
$16.19 \pm$ \\
6.97 \\
\end{tabular} & \begin{tabular}{|c|}
$17.02 \pm$ \\
7.15 \\
\end{tabular} & $\begin{array}{c}14.9 \pm \\
6.5 \\
\end{array}$ & $0.001 *$ \\
\hline Post procedure & $\begin{array}{c}4.95 \pm \\
5.21\end{array}$ & $\begin{array}{l}5.33 \pm \\
5.29\end{array}$ & $\begin{array}{l}4.36 \pm \\
5.04\end{array}$ & $0.048^{*}$ \\
\hline Improvement & \begin{tabular}{|c|}
$-11.24 \pm$ \\
7.85 \\
\end{tabular} & \begin{tabular}{|c|}
$-11.69 \pm$ \\
8.23 \\
\end{tabular} & \begin{tabular}{|c|}
$-10.54 \pm$ \\
7.2 \\
\end{tabular} & 0.122 \\
\hline $\begin{array}{l}\text { Pre-post comparison } \\
\text { (p-value) }\end{array}$ & $<0.001 *$ & $<0.001 *$ & $<0.001 *$ & - \\
\hline
\end{tabular}

Post procedure severe MR was observed in $3.8 \%$ (18) of the patients, which was not statistically significant in both groups with rate of $3.5 \%$ (10/286) vs. $4.3 \%$ $(8 / 184) ; p=0.639$ for multi-track and Inoue balloon group, however, rate of moderate to severe MR was significantly higher for Inoue balloon group $(24.5 \%$
(45/184) vs. $13.6 \%(39 / 286)$; $\mathrm{p}=0.003)$ as compared to multi-track group. Procedure success rate was $98.6 \%$ $(282 / 286)$ vs. $99.5 \%(183 / 184) ; \mathrm{p}=0.378$ for multitrack and Inoue balloon group respectively. One patient in Inoue balloon group and two patients in multi-track group required emergency valve surgery. Stroke was observed in two patients of multi-track group and two patients from the same group developed tamponed. No in-hospital mortality was observed. Other complication such as heart block, bleeding, vascular complications, and congestive heart failure were observed in both groups. Post procedure inhospital complications and outcomes in multi-track and Inoue balloon group are presented in Table 3 .

Table 3: Post procedure in-hospital complications and outcomes stratified by procedure technique

\begin{tabular}{|c|c|c|c|c|}
\hline \multirow{2}{*}{ Outcomes } & \multirow{2}{*}{ Total } & \multicolumn{2}{|c|}{$\begin{array}{l}\text { Procedure } \\
\text { Technique }\end{array}$} & \multirow{2}{*}{ P-value } \\
\hline & & $\begin{array}{l}\text { Multi- } \\
\text { track }\end{array}$ & $\begin{array}{c}\text { Inoue } \\
\text { balloon }\end{array}$ & \\
\hline Total $(\mathbf{N})$ & 470 & 286 & 184 & - \\
\hline \multicolumn{5}{|c|}{ Post-procedure mitral regurgitation } \\
\hline None & $\begin{array}{l}44.9 \% \\
(211)\end{array}$ & $\begin{array}{c}55.2 \% \\
(158) \\
\end{array}$ & $\begin{array}{c}28.8 \% \\
(53)\end{array}$ & $<0.001 *$ \\
\hline Mild & $\begin{array}{c}37.2 \% \\
(175)\end{array}$ & $\begin{array}{c}31.1 \% \\
(89)\end{array}$ & $\begin{array}{c}46.7 \% \\
(86)\end{array}$ & $<0.001 *$ \\
\hline Moderate & $\begin{array}{l}14 \% \\
(66)\end{array}$ & $\begin{array}{c}10.1 \% \\
(29)\end{array}$ & $\begin{array}{c}20.1 \% \\
(37)\end{array}$ & $0.002 *$ \\
\hline Severe & $\begin{array}{c}3.8 \% \\
(18) \\
\end{array}$ & $\begin{array}{c}3.5 \% \\
(10) \\
\end{array}$ & $4.3 \%(8)$ & 0.639 \\
\hline \multicolumn{5}{|c|}{ Post-procedure tricuspid regurgitation } \\
\hline None & $\begin{array}{l}46.4 \% \\
(218) \\
\end{array}$ & $\begin{array}{c}52.4 \% \\
(150) \\
\end{array}$ & $\begin{array}{l}37 \% \\
(68) \\
\end{array}$ & $0.001^{*}$ \\
\hline Mild & $\begin{array}{c}33.8 \% \\
(159)\end{array}$ & $\begin{array}{l}29 \% \\
(83)\end{array}$ & $\begin{array}{l}41.3 \% \\
(76)\end{array}$ & $0.006^{*}$ \\
\hline Moderate & $\begin{array}{c}13.4 \% \\
(63)\end{array}$ & $\begin{array}{c}12.6 \% \\
(36) \\
\end{array}$ & $\begin{array}{l}14.7 \% \\
(27) \\
\end{array}$ & 0.517 \\
\hline Severe & $\begin{array}{c}6.4 \% \\
(30)\end{array}$ & $\begin{array}{l}5.9 \% \\
(17)\end{array}$ & $\begin{array}{c}7.1 \% \\
(13)\end{array}$ & 0.627 \\
\hline \multicolumn{5}{|c|}{ In-hospital outcome } \\
\hline $\begin{array}{l}\text { Successful } \\
\text { procedure }\end{array}$ & $\begin{array}{l}98.9 \% \\
(465)\end{array}$ & $\begin{array}{l}98.6 \% \\
(282)\end{array}$ & $\begin{array}{l}99.5 \% \\
(183)\end{array}$ & 0.378 \\
\hline Stroke/VT/VF & $\begin{array}{l}1.1 \% \\
(5)\end{array}$ & $1.7 \%(5)$ & $0 \%(0)$ & 0.071 \\
\hline Tamponed & $\begin{array}{l}0.4 \% \\
(2)\end{array}$ & $0.7 \%(2)$ & $0 \%(0)$ & 0.256 \\
\hline $\begin{array}{l}\text { Emergency } \\
\text { surgery }\end{array}$ & $\begin{array}{c}0.6 \% \\
(3)\end{array}$ & $0.7 \%(2)$ & $0.5 \%(1)$ & 0.836 \\
\hline
\end{tabular}

\section{DISCUSSION}

This analysis was conducted to compare post procedure severe MR in patients with severe MS after PMBV via Inoue balloon and multi-track double balloon system and observed that rate of postprocedure severe MR was not significantly different between the both techniques (3.5\% (10/286) vs. $4.3 \%$ (8/184); $\mathrm{p}=0.639$ for multi-track and Inoue balloon system respectively) but rate of moderate MR was 
significantly higher in Inoue balloon group (20.1\% vs. $10.1 \% ; \mathrm{p}=0.002$ ). Furthermore, the effectiveness of multi-track systems was observed to be better at improving post-procedure valve area $\left(0.66 \pm 0.31 \mathrm{~cm}^{2}\right.$ vs. $\left.0.56 \pm 0.29 \mathrm{~cm}^{2} ; \mathrm{p}<0.001\right)$ and reducing post procedure left atrio ventricular gradient $(-8.96 \pm 5.01$ $\mathrm{mmHg}$ vs. $-7.79 \pm 4.52 \mathrm{mmHg} ; \mathrm{p}=0.01)$. These differences can be partly explained by the difference in baseline characteristic between the patients managed by both the systems, such as patients in Inoue balloon group had comparatively older age (33.71 \pm 11.43 years vs. $30.57 \pm 10.04$ years; $p=0.002$ ), higher body weight $(55.32 \pm 12.9 \mathrm{~kg}$ vs. $52.14 \pm 12.25 \mathrm{~kg}$; $\mathrm{p}=$ $0.008)$, high Wilkin score ( $7.58 \pm 1.42$ vs. $7.18 \pm 1.46$; $\mathrm{p}=0.004)$, and presence of LA smoke $(25.5 \%$ vs. $11.9 \%$; $\mathrm{p}<0.001)$ at baseline echocardiography. Additionally, the pre-procedure valve area was significantly smaller for the patients managed with Inoue balloon as compared to multi-track system ( 0.93 $\pm 0.2 \mathrm{~cm}^{2}$ vs. $\left.0.98 \pm 0.2 \mathrm{~cm}^{2} ; \mathrm{p}=0.007\right)$. There is a learning curve to the newer technique and operator's confidence in the system is getting stronger and successful PMBV attempts were made in more patients with severe disease (Wilkin score or 10 or 11 and small valve area) through Inoue balloon as compared to multi-track system. Overall procedural success rate was observed to be similar with both the systems $(98.6 \%$ vs. $99.5 \%$; $p=0.378$ for multi-track and Inoue balloon group respectively).

Procedural success rate reported in our patients with both the systems was observed to be better than finings of various other studies from various other parts of the world. Such as, a study conducted by Kang D-H et al. ${ }^{13}$ randomized 302 patients to two techniques Inoue (152) and multi-rack (150) and concluded similar effectiveness of the two techniques with equal successful immediate results ( $84 \%$ vs. $81 \%$ for Inoue balloon and multi-track balloon technique) and MR grade $\geq 3$ in ten patients each in both the groups. Long term median follow-up of 20.7 years outcome of the same patients were reported by the Lee $\mathrm{S}$ et al. ${ }^{14}$ reported composite outcome of mortality, need of surgical correction, repeat PMBV, or deterioration of functional class in 53.9\% of Inoue balloon group vs. $52.7 \%$ of multi-track balloon group and concluded similar effectiveness of both the techniques and achievement of immediate post-PMBV MV area of $\geq$ $1.8 \mathrm{~cm}^{2}$ and commissural MR were reported to be important to optimize long term results of PMBV.

Similarly, Oraby $M$ et al. ${ }^{15}$ reported successful immediate and 18 months outcome of multi-track and Inoue balloon technique with immediate successful results in $94.44 \%$ vs. $95.23 \%$ respectively. Various other investigations have revealed similar immediate-
, short, and long-term hemodynamic and morphologic results of both methods. ${ }^{16-18}$ A study conducted in our population by Asharf $\mathrm{T}$ et al. ${ }^{11}$ reported mild to moderate MR in $53.2 \%$ of the patients after PMBV with multi-track system. Another local study by Farman MT et al. ${ }^{19}$ reported moderate to severe MR after PTMC in 21 (10.7\%) patients out of a total of 197 patients with multi-track system. Data regarding procedural success and post procedural complication for PMBV with Inoue balloon is lacking in our population. Nunes MCP et al. ${ }^{20}$ reported severe MR in $18.6 \%$ after PMBV with most common mechanisms of commissural, commissural with posterior leaflet, leaflets at sub valvular damage or central scallop, and central MR.

This study is first of its kind in our population, however, non-randomized nature of study design and analysis of retrospective data of selected consultant can be potential limitations of this study.

\section{CONCLUSION}

Post-procedure severe mitral regurgitation is a significant and frequent complication. Rate of post procedure sever MR are similar for PMBV via Inoue balloon and multitrack balloon. Both methods are equally effective with equal success rate.

\section{AUTHORS' CONTRIBUTION:}

FF: Conceiving and designing the study, data collection, data analysis, formulation of result and writing the manuscript, and responsible for accuracy of results and integrity of research.

AA, IJB, AM, ASM, SAH, TS, TA, JAS, NK: Conceiving and designing the study, interpretation of data, critical review of manuscript, approval of final draft, and responsible for accuracy of results and integrity of research.

Conflict of interest: Authors declared no conflict of interest.

\section{REFERENCES}

1. Sims Sanyahumbi A, Colquhoun S, Wyber R, Carapetis JR. Global Disease Burden of Group A Streptococcus. 2016 Feb 10. In: Ferretti JJ, Stevens DL, Fischetti VA, editors. Streptococcus pyogenes : Basic Biology to Clinical Manifestations [Internet]. Oklahoma City (OK): University of Oklahoma Health Sciences Center; 2016-. Available from: https://www.ncbi.nlm.nih.gov/sites/books/NBK333415/

2. Carapetis JR, Beaton A, Cunningham MW, Guilherme L, Karthikeyan G, Mayosi BM, et al. Acute rheumatic fever and rheumatic heart disease. Nat Rev Dis Primers. 2016;2(1):1-24

3. Watkins DA, Johnson CO, Colquhoun SM, Karthikeyan G, Beaton A, Bukhman G, et al. Global, Regional, and National Burden of Rheumatic Heart Disease, 1990-2015. New Eng J Med. 2017;377(8):713-22.

4. WHO. Rheumatic heart disease: key facts 2020 [cited 2021 6th March 2021]. Available from: https://www.who.int/newsroom/fact-sheets/detail/rheumatic-heart-disease. 
5. Palafox B, Mocumbi AO, Kumar RK, Ali SK, Kennedy E, Haileamlak A, et al. The WHF roadmap for reducing CV morbidity and mortality through prevention and control of RHD. Glob Heart. 2017;12(1):47-62

6. Zühlke LJ, Beaton A, Engel ME, Hugo-Hamman CT, Karthikeyan G, Katzenellenbogen JM, et al. Group A streptococcus, acute rheumatic fever and rheumatic heart disease: epidemiology and clinical considerations. Curr Treat Options Cardiovasc Med. 2017;19(2):15.

7. Remenyi B, ElGuindy A, Smith Jr SC, Yacoub M, Holmes Jr DR Valvular aspects of rheumatic heart disease. Lancet. 2016;387(10025):1335-46.

8. Zühlke L, Engel ME, Karthikeyan G, Rangarajan S, Mackie P, Cupido B, et al. Characteristics, complications, and gaps in evidence-based interventions in rheumatic heart disease: the Global Rheumatic Heart Disease Registry (the REMEDY study). Eur Heart J. 2015;36(18):1115-22.

9. Otto CM, Nishimura RA, Bonow RO, Carabello BA, Erwin JP, Gentile F, et al. 2020 ACC/AHA Guideline for the Management of Patients With Valvular Heart Disease: A Report of the American College of Cardiology/American Heart Association Joint Committee on Clinical Practice Guidelines. Circulation. 2021;143(5):e72-e227.

10. Nishimura RA, Otto CM, Bonow RO, Carabello BA, Erwin JP, Guyton RA, et al. 2014 AHA/ACC guideline for the management of patients with valvular heart disease: executive summary: a report of the American College of Cardiology/American Heart Association Task Force on Practice Guidelines. J Am Coll Cardiol. 2014;63(22):2438-88

11. Asharf T, Memon MA, Rasool SI, Patel N, Panhwar Z, Farooq F, et al. Preventive strategies to fight complications during percutaneous multitrack balloon valvotomy "refining the technique. J Ayub Med Coll Abbottabad. 2014;26(3):357-60.

12. Elasfar AA, Elsokkary HF. Predictors of developing significant mitral regurgitation following percutaneous mitral commissurotomy with Inoue balloon technique. Cardiol Res Pract. 2011;2011:e703515.

13. Kang D-H, Park S-W, Song J-K, Kim H-S, Hong M-K, Kim J-J, et al. Long-term clinical and echocardiographic outcome of percutaneous mitral valvuloplasty: randomized comparison of Inoue and double-balloon techniques. J Am Coll Cardiol. 2000;35(1):169-75.

14. Lee S, Kang D-H, Kim D-H, Song J-M, Song J-K, Park S-W, et al. Late outcome of percutaneous mitral commissurotomy: randomized comparison of Inoue versus double-balloon technique. Am Heart J. 2017;194:1-8.

15. Oraby M, Youssef A. Immediate and 18-Month Outcome of Balloon Mitral Valvuloplasty: Comparison of Inoue and MultiTrack System. J Interv Cardiol. 2012;25(1):47-52.

16. Youssef AA, Dimitry SR, Soliman H, Abdallah MA. Comparison between Inoue Balloon and Multi-Track Catheter System in Dilatation of Mitral Stenosis Of Similar Echocardiographic Scores. Egypt Heart J. 2009;61(3):363-70

17. Schievano S, Kunzelman K, Nicosia MA, Cochran RP, Einstein DR, Khambadkone S, et al. Percutaneous mitral valve dilatation: single balloon versus double balloon. A finite element study. J Heart Valve Dis. 2009;18(1):28-34.

18. Sharieff S, Aamir K, Sharieff W, Tasneem H, Masood T, Saghir $\mathrm{T}$, et al. Comparison of Inoue Balloon, Metallic Commissurotome and Multi-Track Double-Balloon Valvuloplasty. J Invasive Cardiol. 2008;20:521-5.

19. Farman MT, Khan N, Sial JA, Saghir T, Ashraf T, Rasool SI, et al. Predictors of successful percutaneous transvenous mitral commissurotomy using the Bonhoeffer Multi-Track system in patients with moderate to severe mitral stenosis: Can we see beyond the Wilkins score? Anatol J Cardiol. 2015;15(5):373-9.

20. Nunes MCP, Levine RA, Braulio R, Pascoal-Xavier MA, Elmariah S, Gomes NF, et al. Mitral Regurgitation After Percutaneous Mitral Valvuloplasty: Insights Into Mechanisms and Impact on Clinical Outcomes. JACC Cardiovasc Imaging. 2020;13(12):2513-26.

\section{Address for Correspondence:}

Dr. Faiza Farooq, Senior Registrar at National Institute of Cardiovascular Diseases, Karachi, Pakistan. Email: drffaziz@gmail.com 